\title{
Quarkonium production in hadron collisions at forward rapidity with ALICE at the LHC
}

\author{
Massimiliano Marchisone*, for the ALICE Collaboration \\ Laboratoire de Physique Corpusculaire (LPC), Clermont Université, Université Blaise Pascal, \\ CNRS-IN2P3, Clermont-Ferrand, France \\ Dipartimento di Fisica dell'Università and Sezione INFN, Turin, Italy \\ E-mail: marchiso@to.infn.it
}

\begin{abstract}
Ultrarelativistic heavy-ion collisions produce strongly interacting matter at high temperature and energy density. Under these extreme conditions a deconfined partonic state, called Quark-Gluon Plasma (QGP), is formed.

The measurement of quarkonia (charmonia and bottomonia) is expected to provide essential information about the QGP properties. In pp collisions high precision data serve as crucial test for several competing models of quarkonium hadroproduction and provide the reference for the measurements in AA collisions, while pA collisions are useful to disentangle hot and cold nuclear matter effects.

Within the ALICE Muon Spectrometer, quarkonium production can be measured at forward rapidity $(2.5<y<4)$ and down to $p_{\mathrm{T}}=0$ via the dimuon decay channel.

The latest results on $\mathrm{J} / \psi$ and $\Upsilon$ production in $\mathrm{Pb}-\mathrm{Pb}$ collisions at $\sqrt{s_{\mathrm{NN}}}=2.76 \mathrm{TeV}$ are shown and discussed. An overview of the most important results obtained in $\mathrm{pp}$ and $\mathrm{p}-\mathrm{Pb}$ collisions is also given.
\end{abstract}

The European Physical Society Conference on High Energy Physics - EPS-HEP2013

18-24 July 2013

Stockholm, Sweden

${ }^{*}$ Speaker. 


\section{Introduction}

Among the possible probes of the Quark-Gluon Plasma (a deconfined partonic phase predicted by quantum chromodynamics [1]), heavy quarks are of particular interest since they are expected to be produced in the initial hard partonic scatterings of the heavy-ion collisions and to coexist with the surrounding medium. Therefore, quarkonium (charmonium and bottomonium) measurements provide essential information on the properties of the strongly-interacting medium and, according to the colour-screening model [2], the dissociation probability of the different bound states is expected to provide an estimate of the initial temperature of the system.

The in-medium modification of the quarkonium production is usually quantified through the nuclear modification factor $\left(R_{\mathrm{AA}}\right)$, defined as the ratio of quarkonium yields in heavy-ion collisions and the production cross section in pp collisions scaled by the nuclear overlap function $\left(T_{\mathrm{AA}}\right.$, number of binary collisions divided by the nucleon-nucleon inelastic cross section) obtained from a Glauber model calculation [3]:

$$
R_{\mathrm{AA}}=\frac{Y_{\mathrm{AA}}}{\left\langle T_{\mathrm{AA}}\right\rangle \cdot \sigma_{\mathrm{pp}}} .
$$

The interpretation of the measurements is complicated by several factors: the feed-down contributions from higher-mass resonances into the observed quarkonium yield, the B-hadrons decay into charmonium and the quarkonium (re)generation due to the q $\bar{q}$ recombination (expected to be important for c̄̄ pairs at LHC energies) [4].

Other important mechanisms are the cold nuclear matter (CNM) effects, such as nuclear shadowing of the parton distribution functions or coherent parton energy loss, which can break the scaling with $\left\langle T_{\mathrm{AA}}\right\rangle$ even in absence of the QGP [5]. Data from proton-nucleus (pA) collisions are used to quantify these CNM effects.

ALICE [6] is the LHC experiment dedicated to the study of heavy-ion collisions. At forward rapidity $(2.5<y<4)$ the quarkonium production can be measured with the Muon Spectrometer down to $p_{\mathrm{T}}=0$. It consists of a ten interaction length thick front absorber filtering the muons upstream of five tracking stations comprising two planes of cathode pad chambers each, with the third station inside a dipole magnet with a $3 \mathrm{~T} \cdot \mathrm{m}$ field integral. The tracking apparatus is completed by a triggering system made of four planes of resistive plate chambers downstream of a $1.2 \mathrm{~m}$ thick iron wall. The iron wall allows for an efficient rejection of light hadrons escaping from the front absorber and a part of the low-momentum muons, mainly from $\pi$ and $\mathrm{K}$ decays. Both tracking chambers and trigger chambers are protected by a beam shield stretching along the whole detector length.

\section{Results in pp collisions}

The ALICE experiment collected data in pp collisions at various energies as a reference for heavy-ion studies and for pp-specific measurements.

The cross section of inclusive (prompt and non-prompt) $\mathrm{J} / \psi$ was measured in pp collisions at centre-of-mass energies of 2.76 and $7 \mathrm{TeV}$ at forward rapidity [7]. The transverse momentum $\left(p_{\mathrm{T}}\right)$ dependence is well predicted by the NRQCD approach at both energies [8] as demonstrated in the left panel of Fig. 1. The results at $2.76 \mathrm{TeV}$ were used as pp reference for the $R_{\mathrm{AA}}$ evaluation. 

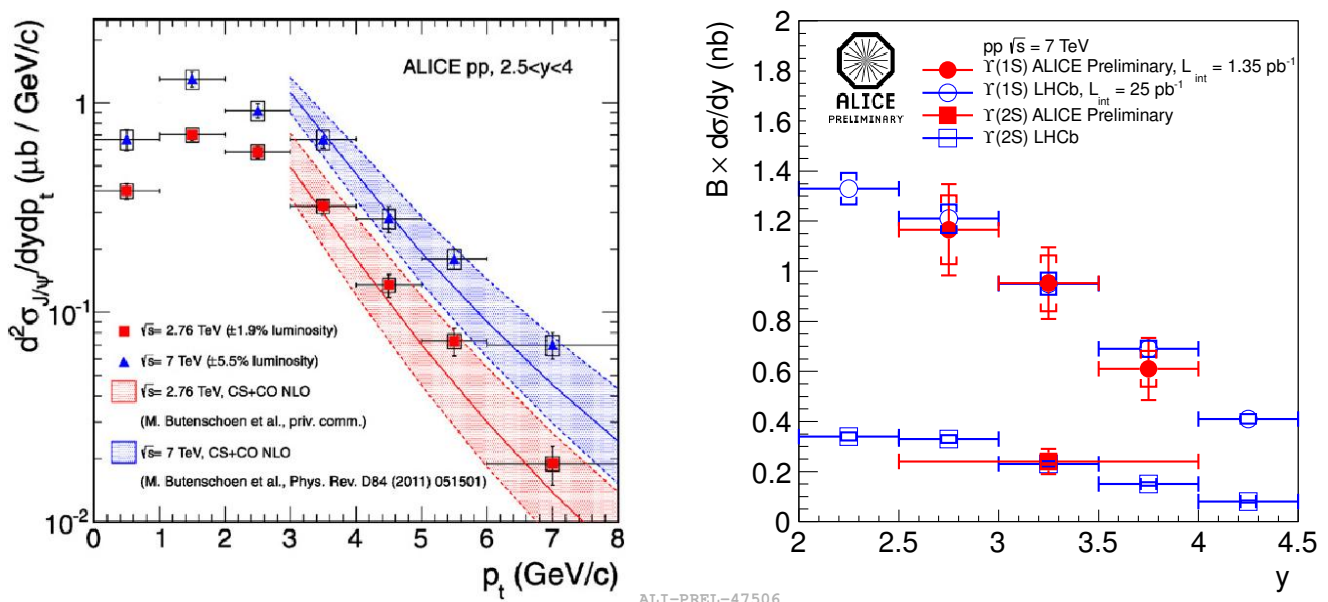

Figure 1: Left: inclusive $\mathrm{J} / \psi$ cross sections vs $p_{\mathrm{T}}$ in pp collisions at 2.76 and $7 \mathrm{TeV}$ with NRQCD predictions [8]. Right: $\Upsilon(1 S)$ and $\Upsilon(2 S)$ cross sections vs rapidity at $7 \mathrm{TeV}$ measured by ALICE and LHCb [9].

The $\Upsilon(1 S)$ and $\Upsilon(2 S)$ production cross sections at $\sqrt{s}=7 \mathrm{TeV}$ were measured as a function of rapidity (Fig. 1, right) and of $p_{\mathrm{T}}$ (not shown here). The comparison with the LHCb results [9] shows a good consistency.

\section{Results in $\mathbf{P b}-\mathbf{P b}$ collisions}

The inclusive $\mathrm{J} / \psi$ and $\Upsilon(1 S)$ nuclear modification factors were studied in $\mathrm{Pb}-\mathrm{Pb}$ collisions at a center-of-mass energy of $2.76 \mathrm{TeV}$ per nucleon in the rapidity range $2.5<y<4$ and $p_{\mathrm{T}}>0$.
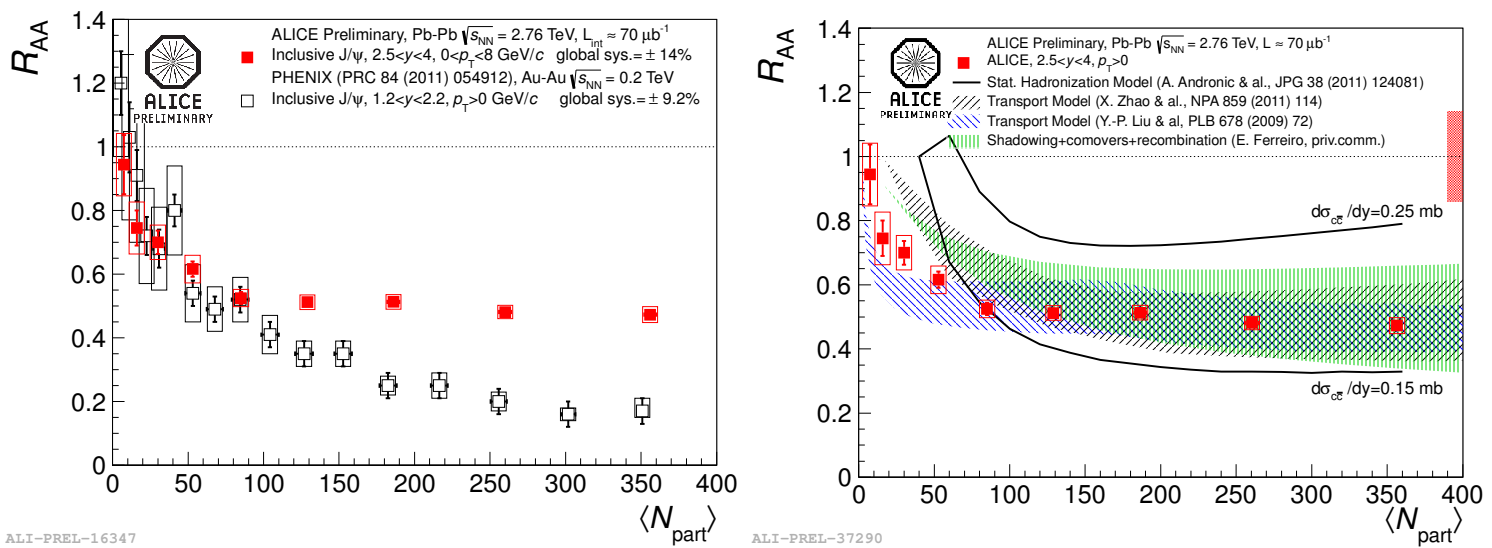

Figure 2: Inclusive $\mathrm{J} / \psi$ nuclear modification factor as a function of the number of participants compared to PHENIX measurements at RHIC (left) and to theoretical models (right).

In the left panel of Fig. 2 the $\mathrm{J} / \psi R_{\mathrm{AA}}$ centrality dependence is compared to the results of the PHENIX experiment [10]. At LHC energies the suppression saturates moving towards central collisions where the $\mathrm{J} / \psi$ is about three times less suppressed than at RHIC. The larger $R_{\mathrm{AA}}$ at the LHC is consistent with the effect of $\mathrm{J} / \psi$ regeneration by recombination of initially uncorrelated 
$\mathrm{c}$ and $\overline{\mathrm{c}}$ quarks, which counteracts the suppression by colour screening. The right panel of Fig. 2 shows the predictions within partonic transport models $[11,12,13]$ and the statistical hadronization model [14], compared with the data.

Furthermore, the $\mathrm{J} / \psi$ suppression increases with the transverse momentum in central collisions, while the dependence is negligible in the peripheral ones as shown in Fig. 3 (left). A reasonable description of the data is provided by the models that include a $p_{\mathrm{T}}$-dependent contribution of recombination amounting, at low $p_{\mathrm{T}}$, to $60 \%$ in central and $30 \%$ in peripheral collisions $[11,12]$.

Finally, the increase of the suppression towards larger rapidity is not described by the expected rapidity dependence of nuclear shadowing, as demonstrated in Fig. 3 (right).
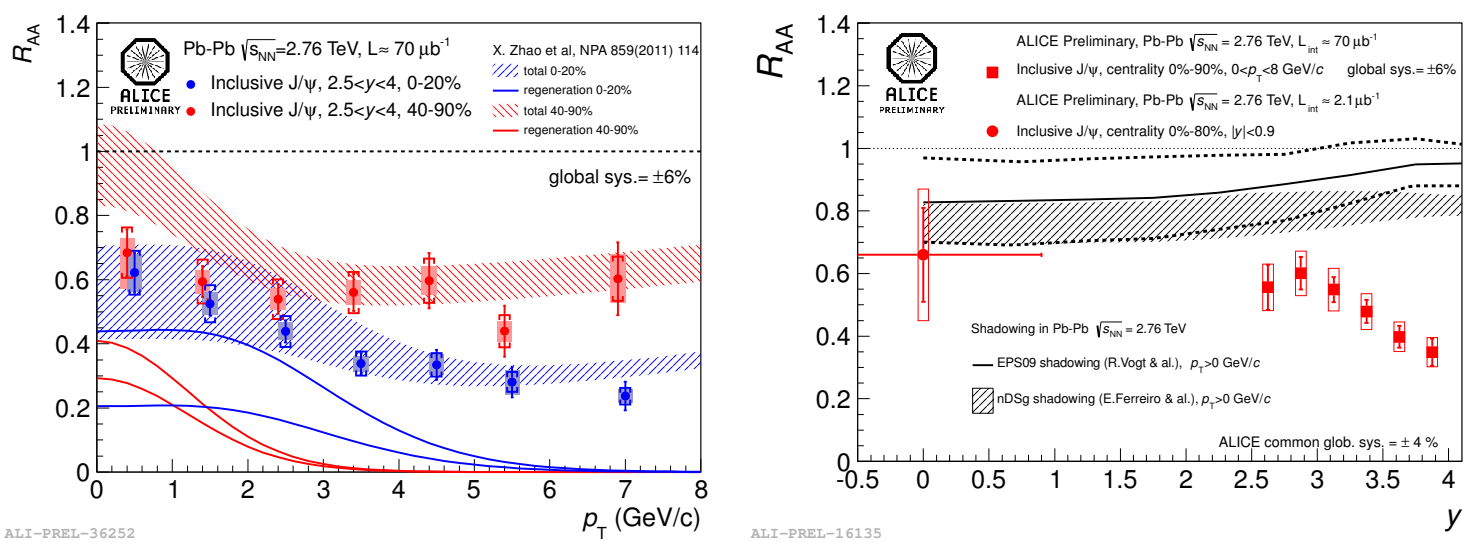

Figure 3: Left: transverse momentum dependence of the inclusive $\mathrm{J} / \psi R_{\mathrm{AA}}$ for two bins of centrality compared to transport model predictions. Right: inclusive $\mathrm{J} / \psi R_{\mathrm{AA}}$ as a function of rapidity compared to shadowing calculations. The point at midrapidity is obtained exploiting the $\mathrm{J} / \psi$ dielectron decays in the ALICE central barrel.

In heavy-ion collisions, the $\Upsilon(1 S)$ state is expected to dissociate at a higher temperature than all the other quarkonia. In addition, the $b \bar{b}$ production cross section is smaller than the cē one, resulting in a lower probability of bottomonium regeneration compared to charmonium. Therefore the $\Upsilon$ resonances can be considered as an effective thermometer of the system [15].

The inclusive $\Upsilon(1 S) R_{\mathrm{AA}}$ measured at forward rapidity and for $p_{\mathrm{T}}>0$ is shown in Fig. 4 as a function of the average number of participating nucleons $\left(\left\langle N_{\text {part }}\right\rangle\right)$ on the left and as a function of rapidity on the right, along with the CMS measurements at $|y|<2.4$ [16]. The first panel indicates a clear suppression, increasing with towards more central collisions. It might be compatible with the expectations based on the melting of the excited bottomonium states, which contribute through feed-down to about 50\% of the observed $\Upsilon(1 S)$ [17]. The suppression is similar at forward and midrapidity for both central and semi-peripheral collisions.

In the left panel of Fig. 5, the comparison with a model based on a hydrodynamic formalism [18] suggests the smallest possible value of shear viscosity over entropy density $(\eta / s)$. However, it does not take into account any cold nuclear matter effect nor $\Upsilon(1 S)$ regeneration. In the rate equation model [19] CNM effects are considered by an overall absorption cross section from 0 to $2 \mathrm{mb}$ including shadowing, Cronin effect and nuclear absorption. A small amount of $b \bar{b}$ recombination is also included in the model. Also in this case the predictions are consistent with the data. 

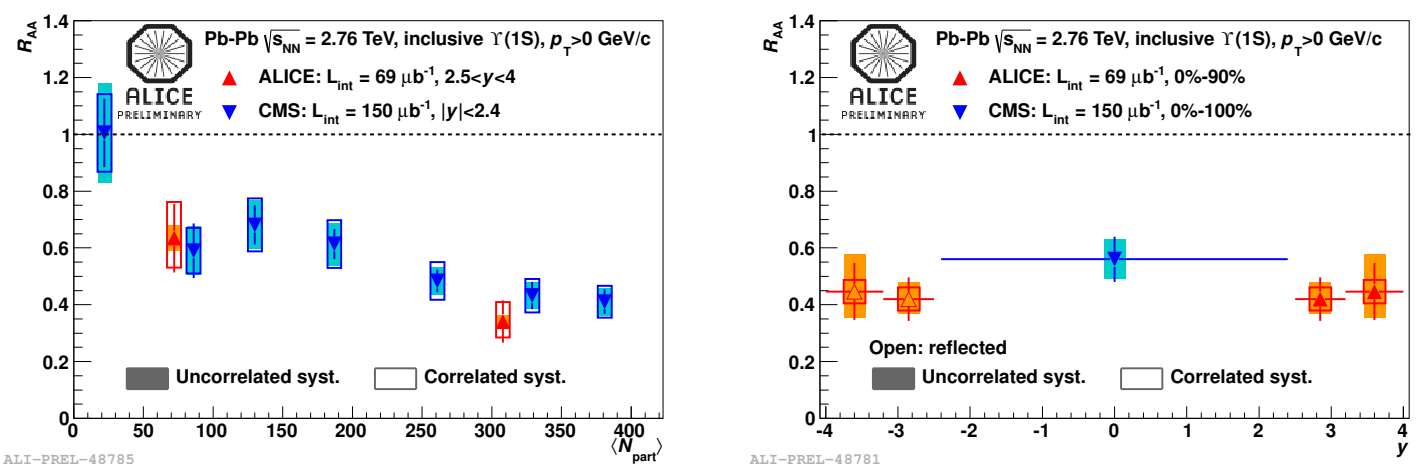

Figure 4: ALICE and CMS nuclear modification factor of the inclusive $\Upsilon(1 S)$ production as a function of the average number of participating nucleons (left) and rapidity (right).
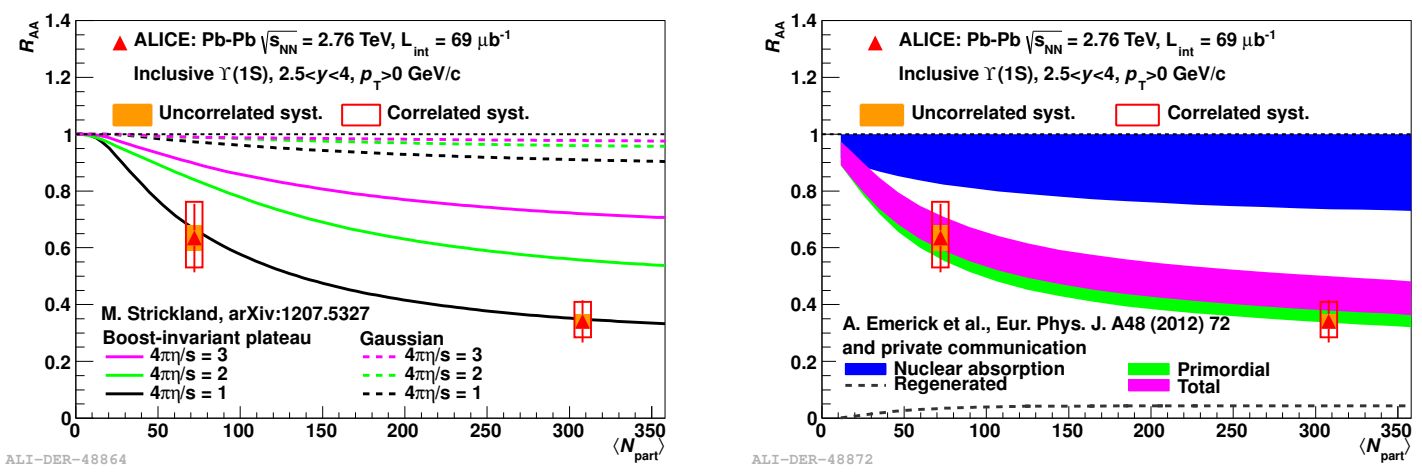

Figure 5: Inclusive $\Upsilon(1 S) R_{\mathrm{AA}}$ as a function of the average number of participants compared to a hydrodynamic model on the left and to a rate equation model on the right.

\section{Results in $\mathbf{p}-\mathbf{P b}$ collisions}

In order to quantify the size of hot nuclear matter effects, it is extremely important to evaluate how cold nuclear matter modifies the $\mathrm{J} / \psi$ and the $\Upsilon(1 S)$ production. The effects are quantified through the nuclear modification factor $R_{\mathrm{pA}}$, similar to the $R_{\mathrm{AA}}$ defined for nucleus-nucleus collisions. In order to do so, ALICE collected data in $\mathrm{p}-\mathrm{Pb}$ and $\mathrm{Pb}-\mathrm{p}$ collisions at $\sqrt{s_{\mathrm{NN}}}=5.02 \mathrm{TeV}$. Due to the asymmetric beams circulating in the LHC, in the following figures the positive rapidity region corresponds to the data collected with the proton beam going towards the ALICE Muon Spectrometer, while the negative rapidity region corresponds to the $\mathrm{Pb}$ beam towards the Muon Spectrometer.

Lacking pp collisions at $\sqrt{s}=5.02 \mathrm{TeV}$ at the LHC, the cross section references are obtained interpolating previous results at higher and lower energies [20].

First results on the inclusive $\mathrm{J} / \psi R_{\mathrm{pA}}$ measured by ALICE in the rapidity ranges $-4.46<$ $y_{\mathrm{cms}}<-2.96$ and $2.03<y_{\mathrm{cms}}<3.53$ are shown in Fig. 6 (left) as a function of rapidity [20]. A suppression of the $\mathrm{J} / \psi$ yield is observed at forward rapidity, while no suppression is visible in the backward region. The results are reasonably described by models based on nuclear shadowing [21] and partonic energy loss with or without shadowing [22, 23]. Color Glass Condensate (CGC) 
predictions [24], also shown, overestimate the observed suppression.

The $R_{\mathrm{FB}}$ factor defined as the ratio between forward and backward $\mathrm{J} / \psi$ yields has been introduced to guarantee a better accuracy, since it does not depend on the pp reference. This variable has to be studied in the common kinematic interval covered in $|y|$ and it is therefore limited to the range $2.96<y_{\mathrm{cms}}<3.53$ (Fig. 6, right). Also in this case, models are consistent with the data, even if the theoretical and experimental uncertainties prevent from drawing more precise conclusions on the role of the different contributions [20].
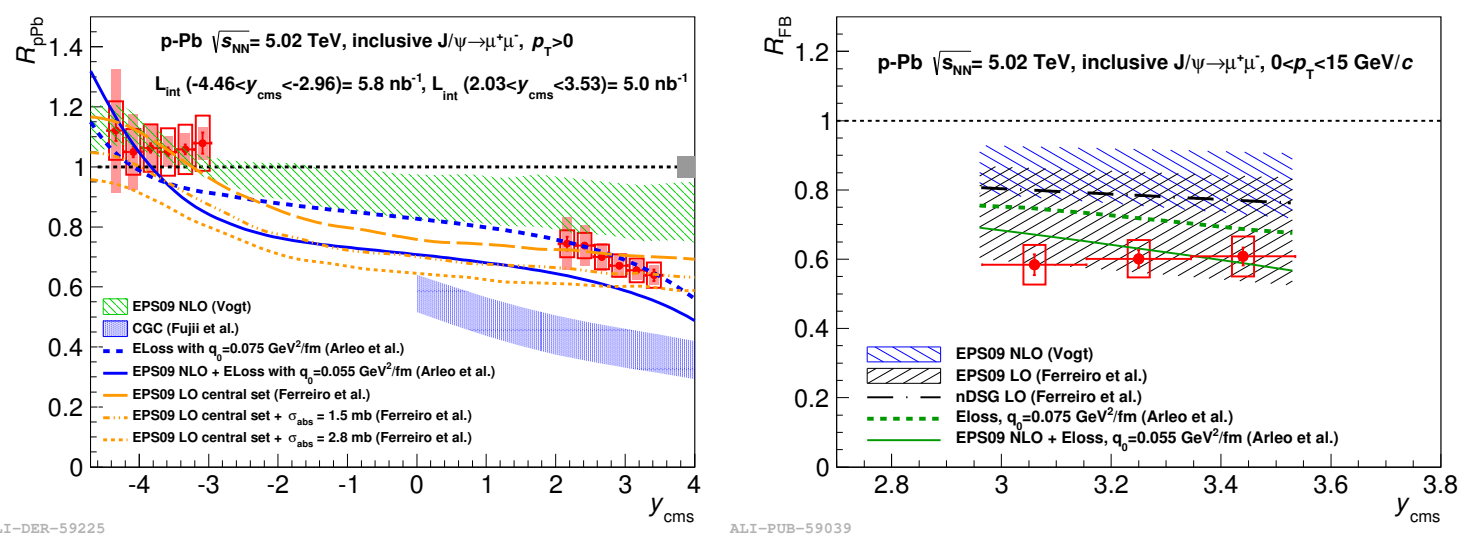

Figure 6: $\mathrm{J} / \psi R_{\mathrm{pA}}$ (left) and $R_{\mathrm{FB}}$ (right) as a function of rapidity compared to theory predictions.

The $R_{\mathrm{pA}}$ for the $\Upsilon(1 S)$ meson is shown as a function of centrality in Fig. 7 along with the $\mathrm{J} / \psi$ results described above. The measured suppression patterns of the two mesons are identical at positive rapidity and similar within large uncertainties at backward $y$. Theoretical predictions for both quarkonium states based on shadowing effects [25] on the left and on energy loss mechanism [22] on the right are shown. Other calculations are also available [21].
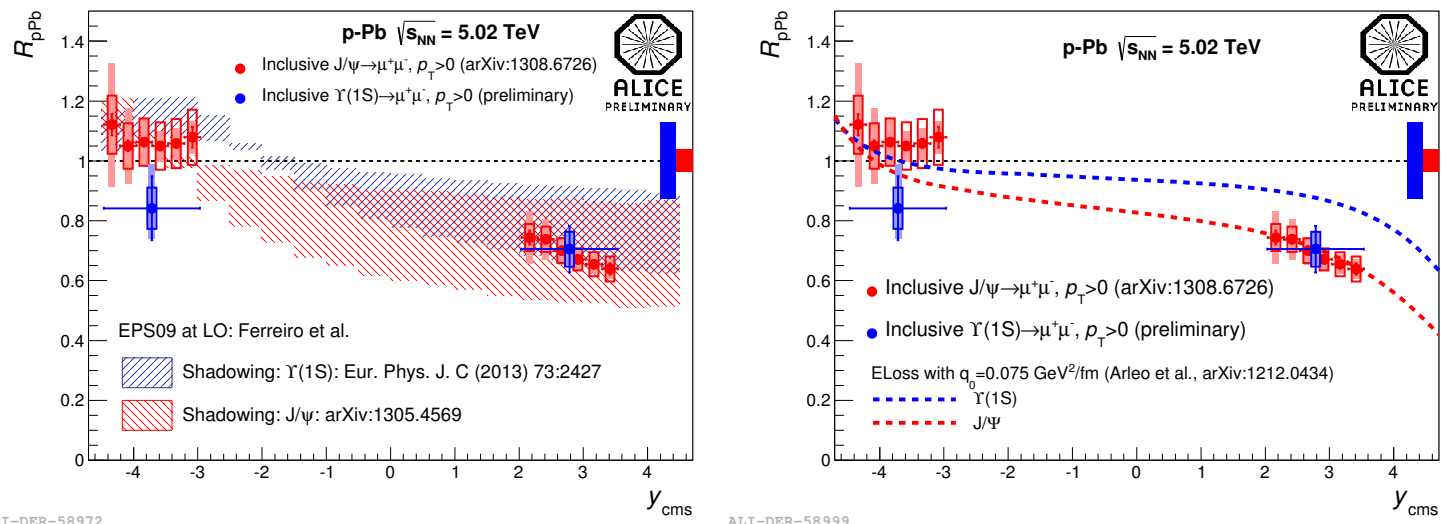

Figure 7: Inclusive $\Upsilon(1 S)$ and $\mathrm{J} / \psi$ nuclear modification factors as a function of rapidity compared to models including shadowing (left) and parton energy loss (right).

\section{Conclusions}

The ALICE Collaboration measured the quarkonium production at forward rapidity and down 
to $p_{\mathrm{T}}=0$ in $\mathrm{pp}, \mathrm{Pb}-\mathrm{Pb}$ and $\mathrm{p}-\mathrm{Pb}$ collisions.

The $\mathrm{J} / \psi$ cross sections in pp collisions are well reproduced by NRQCD models.

Both quarkonium states are suppressed in heavy-ion collisions. For charmonium, an important cī recombination effect could be present, as suggested by the $p_{\mathrm{T}}$ dependence of the $R_{\mathrm{AA}}$ and by the comparison with RHIC results. For $\Upsilon(1 S)$, the comparison of data from ALICE and CMS shows that the rapidity dependence of the suppression is weak.

Finally, in $\mathrm{p}-\mathrm{Pb}$ collisions, models including nuclear shadowing and energy loss in cold nuclear matter provide a good description of the $p_{\mathrm{T}}$-integrated nuclear modification factor of $\mathrm{J} / \psi$ as function of rapidity and of $\Upsilon(1 S)$ at forward rapidity, while they overestimate the $\Upsilon(1 S) R_{\mathrm{pA}}$ at backward rapidity.

\section{References}

[1] S. Hands, Contemp. Phys. 42 (2001) 209

[2] T. Matsui and H. Satz, Phys. Lett. B 178 (1986) 416

[3] K. Aamodt et al. (ALICE Collaboration), Phys. Rev. Lett. 106 (2011) 032301

[4] E. L. Bratkovskaya et al., Phys. Rev. C 69 (2004) 054903

[5] R. Vogt, Phys. Rev. C 81 (2010) 044903

[6] K. Aamodt et al. (ALICE Collaboration), JINST 3 (2008) S08002

[7] B. Abelev et al. (ALICE Collaboration), Phys. Lett. B 718 (2012) 2

[8] M. Butenschoen et al., Phys. Rev. D 84 (2011) 051501 and priv. comm.

[9] R. Aaij et al. (LHCb Collaboration), EPJ C 72 (2012) 2025

[10] A. Adare et al. (PHENIX Collaboration), Phys. Rev. C 84 (2011) 054912

[11] X. Zhao et al., Nucl. Phys. A 859 (2011) 114 and priv. comm.

[12] Y.-P. Liu et al., Phys. Lett. B 678 (2009) 72 and priv. comm.

[13] E. G. Ferreiro, arXiv:1210.3209

[14] A. Andronic et al., J. of Phys. G 38 (2011) 124081

[15] S. Digal et al., Phys. Rev. D 64 (2001) 094015

[16] S. Chatrchyan et al. (CMS Collaboration), Phys. Rev. Lett. 109 (2012) 222301

[17] T. Affolder et al. (CDF Collaboration), Phys. Rev. Lett. 84 (2000) 2094

[18] M. Strickland, Phys. Rev. Lett. 107 (2011) 132301

[19] A. Emerick et al., Eur. Phys. J. A 48 (2012) 72

[20] B. Abelev et al. (ALICE Collaboration), arXiv:1308.6726

[21] J. L. Albacete et al., Int. J. Mod. Phys. E 22 (2013) 1330007

[22] F. Arleo et al., arXiv:1212.0434

[23] E. G. Ferreiro, arXiv:1305.4569

[24] H. Fuji et al., arXiv:1304.2221

[25] E. G. Ferreiro et al., Eur. Phys. J. C 73 (2013) 2427 